\title{
Análisis transiente de la demanda de agua uniformemente distribuida
}

\section{Transient analysis of the uniformly distributed water demand}

Fecha de entrega: 9 de enero 2019

Fecha de aceptación: 8 de noviembre 2019

\section{John Twyman}

Twyman Ingenieros Consultores, Pasaje Dos \# 362, Rancagua, Chile, john@twyman.cl

Se analiza el flujo transitorio en redes de tuberías generado por demandas uniformemente distribuidas (DUDs). Se muestra un algoritmo numérico original, basado en el Método de las Características (MC), que permite incluir el enfoque de la DUD insensible a la presión en los nodos internos de una tubería sin modificar su discretización original. Los resultados obtenidos por la DUD se comparan con el enfoque que distribuye el $50 \%$ de la demanda total de agua en los extremos aguas arriba y aguas abajo de la tubería. La conclusión general es que el enfoque de la DUD es útil para conocer el comportamiento transitorio de una tubería cuando cada uno de sus nodos internos tiene una demanda de agua, aunque su formulación sea preliminar y requiera algunas extensiones que le permitan abordar el problema de manera más adecuada.

Palabras claves: golpe de ariete, método de las características $M C$, demanda uniformemente distribuida DUD
Transient flow generated in pipe networks by uniformly distributed demands (UDDs) is analyzed. An original numerical algorithm based on the Method of Characteristics (MOC) is shown, which allows including the pressure-insensitive UDD's approach at the pipe internal nodes without modifying its original discretization. The results delivered by the UDD's approach are compared with the approach that distributes $50 \%$ of the total water demand in the pipe's upstream and downstream ends. The general conclusion is that the UDD's approach is useful to know the pipe transient behaviour when every one of its internal nodes have a water demand, although its formulation is preliminary and it requires some extensions that allow it to approach the problem in more suitable way.

Keywords: water hammer, method of characteristics $M O C$, uniformly distributed demand UDD

\section{Introducción}

Existe escasa literatura que aborde la simulación, en estado de flujo transiente, de una demanda uniformemente distribuida (DUD). Por ejemplo, McInnis y Karney (1995) investigan los efectos de diversos modelos de demandas en orden a predecir el comportamiento hidráulico de los sistemas, destacando un modelo de distribución uniforme del consumo en tramos seleccionados que funciona en base al Método de las Características MC. En este modelo los sistemas, que sirven principalmente a zonas residenciales de baja densidad, pueden ser aproximados mediante un flujo lateral uniforme a lo largo de todo o parte de la tubería. La formulación se basa en una extensión de la ecuación de continuidad para el flujo transiente mediante la adición de un término que depende de las características de la tubería (velocidad de la onda y sección transversal) y de una demanda lateral uniforme que sale del tramo. Según los autores, el modelo permite predecir adecuadamente el descenso inicial de la presión y el primer máximo de sobrepresión positiva al simular el apagado de una motobomba, aunque reconocen que su formulación es inadecuada para representar el decaimiento del transiente a medida que avanza el tiempo de simulación. Jung et al. (2007) indican que el proceso de esqueletonización de una red, es decir, la selección de aquellas partes que tienen un impacto significativo en su comportamiento hidráulico (Shamrukh, 2005), obliga a relocalizar las demandas cuando los nodos internos de algunas tuberías son eliminadas del modelo, lo cual debe realizarse con sumo cuidado al realizar un análisis del flujo transiente en redes de tuberías porque dicho procedimiento podría afectar la disipación y reflexión de las ondas de presión. Tsakiris y Spiliotis (2012) indican que el uso de una demanda uniformemente distribuida es válido sólo cuando hay 
desconocimiento sobre las demandas y conexiones reales en una red, y que la aproximación mediante demandas nodales, aun siendo menos realista, facilita el análisis de las redes de tuberías. Izquierdo et al. (2013) indican que los modelos que simulan las variables de estado en grandes redes de tuberías usualmente dejan de lado las demandas intermedias, siendo la regla aplicar el Método de Repartición Media MRM que busca localizarlas en los nodos extremos de las líneas: 50\% en el nodo ubicado aguas arriba de la línea, 50\% en el nodo ubicado aguas abajo. Este método funciona siempre y cuando haya inexistencia de concentraciones de demanda en la línea que representen un porcentaje importante de su caudal total. Otros autores (Mohammed y Gad, 2012; Gad y Mohammed, 2014) plantean la importancia que tiene la simplificación de las redes durante las primeras etapas de diseño, especialmente lo referente a la concentración de las demandas en los nodos de la red. Al analizar el flujo transiente rápido debido a una demanda distribuida y a otra concentrada en los nodos de dos redes distintas, concluyen que la simplificación basada en la demanda concentrada en los nodos incrementa la presión transiente, y que este tipo de aproximación debiera ser aplicada con mucho cuidado, ya que la suposición de que la demanda total a lo largo de una tubería puede ser representada en la forma de dos consumos ubicados en sus nodos terminales puede ser una fuente de errores en el cálculo de la pérdida de carga, lo cual puede afectar en forma significativa los análisis de calibración, diseño, rehabilitación u operación de los sistemas (Kanakoudis y Gonelas, 2014). Esto lleva a concluir que la asignación de las demandas en los nodos constituye uno de los pasos más relevantes en el análisis de redes de tuberías (Rao et al., 2015), lo cual es inaplicable cuando en la red existen tramos con demandas uniformemente distribuidas DUDs que, debido a su importancia, disposición o función dentro del sistema, deben ser excluidas de la aplicación del MRM.

\section{Material y métodos}

La Figura 1 muestra una tubería ejemplo de longitud $L$ que está dividida en $N=5$ subtramos de igual longitud $\Delta x, \mathrm{y}$ que tiene una demanda $q$ en sus nodos internos 2, 3, 4 y 5, la cual está uniformemente distribuida. El procedimiento usual de discretización considera a la tubería anterior como una serie de 5 tramos con demandas $q$ en alguno de los nodos de borde de las tuberías 1, 2, 3, 4 y 5. Esto significa considerar 5 tramos de longitud $\Delta x$ cada uno, de modo de que cada condición $q$ pueda corresponder a un nodo de borde específico. Si la tubería de la Figura 1 pudiera trabajar con $q$ en sus nodos internos sin necesidad de modificar la discretización inicial, entonces al dividirla con $N=5$ obtenemos el siguiente paso de tiempo acorde al número de Courant $C_{\mathrm{n}}: \Delta t=L /(N a)=L /(5 a)$, donde $\Delta t$ es el paso de tiempo en s y $a$ es la velocidad de la onda en $\mathrm{m} / \mathrm{s}$.

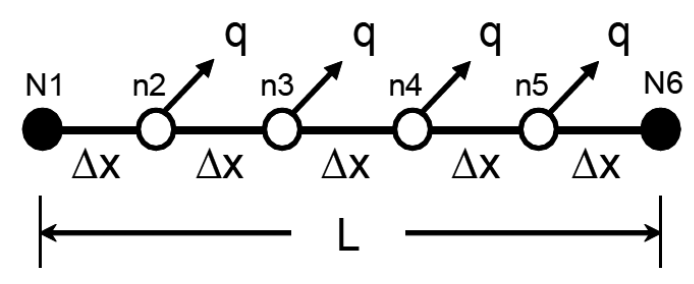

Figura 1: Esquema de una tubería ubicada entre los nodos de borde N1 y N6, subdividida en 5 subtramos y con una DUD $(q)$ entre los nodos internos n2 y n5 ( $L$ : longitud del tramo)

Sin embargo, de ser imposible trabajar con $q$ como parte de una condición de borde global, entonces el análisis transiente exigiría que la tubería fuera dividida en 5 tramos de longitud $\Delta x$ cada uno. En este caso, de optar por $N=5$ para el tramo más corto, el paso de tiempo es: $\Delta t^{*}=\Delta x /(5 a)$. Como $\Delta x$ corresponde a la quinta parte de $L$, obviamente que $\Delta t^{*}<<\Delta$, ralentizando la solución y exigiendo más recursos de memoria computacional a la simulación. En términos numéricos, si la tubería tiene una longitud $L=1000 \mathrm{~m}, a=1000 \mathrm{~m} / \mathrm{s}$ y es subdividida con $N$ $=5$, y puede ser resuelta tomando en cuenta una DUD de magnitud $q$, sin incorporar nuevas condiciones de borde, entonces $\Delta t=1000 /(5 \cdot 1000)=0.2 \mathrm{~s}$. En caso contrario, de carecer de una formulación para abordar la DUD de magnitud $q$, y considerando un $N=5$ para el tramo más corto, entonces $\Delta t=200 /(5 \cdot 1000)=0.04 \mathrm{~s}$. En este caso $\Delta t^{*}$ es un $80 \%$ menor que $\Delta t$, y el $N$ total pasa de 5 (caso con solución para la DUD) a $N=25$ (caso sin solución para la DUD), con un incremento del $400 \%$, con todas las implicancias que esto puede representar en términos de uso de recursos computacionales. El objetivo del presente artículo es plantear una solución al caso mostrado en la Figura 1 utilizando el MC, sin necesidad de modificar la discretización inicial del tramo. La teoría básica sobre el golpe de ariete (ecuaciones) puede estudiarse en Ghidaoui et al. (2005). El MC y el cálculo de a son mostrados en profundidad en algunos textos clásicos (e.g. Wylie y 
Streeter, 1978, 1993; Chaudhry, 1968, 1979; Watters, 1979; 1984), y también en textos y artículos recientes (e.g. Chaudhry, 2014; Twyman, 2016a, 2016b, 2017, 2018), por lo que se recomienda su lectura para profundizar conocimientos. Sin embargo, dada su importancia en el modelo que se propondrá en una sección posterior, se prestará más atención a la forma de tratar un nodo simple en el contexto del $\mathrm{MC}$, aspecto que será tratado brevemente a continuación.

\section{Tratamiento de un nodo simple}

Un nodo simple (Figura 2) es una condición de borde que contiene sólo tuberías que descargan hacia (o desde) el nodo de la red (Karney y McInnis, 1992). Si las pérdidas de carga son insignificantes en el nodo de la red, entonces es posible suponer que la cota piezométrica en el nodo puede representarse por un valor único Hp. Para las tuberías que descargan hacia el nodo es válida la ecuación característica positiva, y para las tuberías que descargan desde el nodo es válida la ecuación característica negativa (Ghidaoui et al., 2005), lo cual significa que:

$$
\begin{aligned}
& -\mathrm{H}_{\mathrm{P}}\left[\sum_{\mathrm{k} \in \mathrm{N} 1} \frac{\mathrm{gA}(\mathrm{k})}{a(\mathrm{k})}+\sum_{\mathrm{j} \in \mathrm{N} 2} \frac{\mathrm{gA}(\mathrm{j})}{a(\mathrm{j})}\right]+\sum_{\mathrm{k} \in \mathrm{N} 1} \frac{\mathrm{gA}(\mathrm{k})}{a(\mathrm{k})} \mathrm{CP}(\mathrm{k})+ \\
& \sum_{\mathrm{j} \in \mathrm{N} 2} \frac{\mathrm{gA}(\mathrm{j})}{a(\mathrm{j})} \mathrm{CM}(\mathrm{j})-\mathrm{Qext}=0
\end{aligned}
$$

Donde N1 y N2 son el conjunto de tuberías que descargan hacia (o desde) el nodo de la red, respectivamente. Qext es el flujo nodal conocido en $\mathrm{m}^{3} / \mathrm{s}$, + si se descarga desde el nodo de la red, - en caso contrario, g es la constante de gravedad en $\mathrm{m} / \mathrm{s}^{2}, \mathrm{~A}(\mathrm{k})$ y $\mathrm{A}(\mathrm{j})$ son la sección transversal de las tuberías $\mathrm{k}$ (que descargan hacia el nodo) y $\mathrm{j}$ (que descargan desde el nodo) en $\mathrm{m}^{2}, a(\mathrm{k})$ y $a(\mathrm{j})$ son la velocidad de la onda en $\mathrm{m} / \mathrm{s}$ de las tuberías $\mathrm{k} \mathrm{y} \mathrm{j}$, respectivamente. $\mathrm{CP}(\mathrm{k})$ y $\mathrm{CM}(\mathrm{j})$ son constantes conocidas y relacionadas con las curvas características positiva y negativa, respectivamente en $m$.

La ecuación (1) representa una expresión general para un nodo simple que permite simplificar el tratamiento topológico de las redes complejas (Wylie, 1986; Salgado et al., 1992), y cuyo planteamiento y desarrollo completo en detalle ha sido descrito por algunos autores (Karney,
1984; Karney y McInnis, 1992).

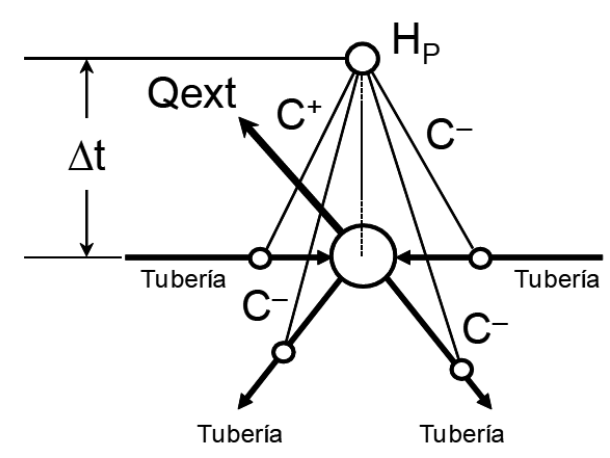

Figura 2: Esquema de un nodo simple

\section{Modelación de demandas uniformemente distribuidas DUDs}

Haciendo una analogía con la ecuación (1), si el almacenamiento en el nodo interno $\mathrm{P}$ de una tubería es nulo, entonces se debe cumplir la ecuación de continuidad válida para el estado de flujo permanente, la cual establece que la suma algebraica de los flujos de descarga hacia (o desde) el nodo debe ser igual a cero, es decir, se debe cumplir la siguiente relación a partir de la ecuación (1), descartando las sumatorias y considerando que $\mathrm{gA}(\mathrm{k}) / a(\mathrm{k})$ $=\mathrm{gA}(\mathrm{j}) / a(\mathrm{j})=\mathrm{gA} / a$ y Qext $=q$ :

$$
-\frac{\mathrm{gA}}{a} \mathrm{Hpi}+\frac{\mathrm{gA}}{a} \mathrm{CP}-\frac{\mathrm{gA}}{a}[\mathrm{Hpi}-\mathrm{CM}]-q=0
$$

con $\mathrm{i}=2, \ldots, N$. Despejando Hpi se obtiene lo siguiente:

$$
\mathrm{Hpi}=\frac{\mathrm{CP}+\mathrm{CM}}{2}-\left(\frac{a}{\mathrm{gA}} \frac{\mathrm{q}}{2}\right)
$$

con: $\mathrm{CP}=\mathrm{H}_{\mathrm{i}-1}+\mathrm{c} \mathrm{Q}_{\mathrm{i}-1}-\mathrm{R} \mathrm{Q}_{\mathrm{i}-1}\left|\mathrm{Q}_{\mathrm{i}-1}\right|, \mathrm{CM}=\mathrm{H}_{\mathrm{i}+1}-\mathrm{c} \mathrm{Q}_{\mathrm{i}+1}-\mathrm{R}$ $\mathrm{Q}_{\mathrm{i}+1}\left|\mathrm{Q}_{\mathrm{i}+1}\right|, \mathrm{R}=f \Delta \mathrm{x} /\left(2 \mathrm{gDA}^{2}\right), \mathrm{c}=a / \mathrm{gA}, f=$ factor de fricción (Darcy) y $\mathrm{D}$ es el diámetro interno de la tubería. Conocido Hpi, el valor del caudal nodal puede obtenerse a partir de las fórmulas $\mathrm{Qpi}=(\mathrm{gA} / a)(\mathrm{CP}-\mathrm{Hpi})$ o $\mathrm{Qpi}=(\mathrm{gA} / a)(\mathrm{Hpi}$ - CM). La Figura 3 muestra un esquema del nodo interno con demanda $q$.

En resumen, la ecuación (3) permite calcular las presiones generadas por una DUD de tamaño $q$, lo cual permite resolver la variable de estado Hpi en los nodos internos de una tubería sin necesidad de tener que re-discretizar 
la red a partir del tramo más corto, de tamaño $\Delta x$, que aparecería cada vez que haya que considerar al nodo con una demanda $q$ como una condición de borde externa para efectos de discretización de la red.

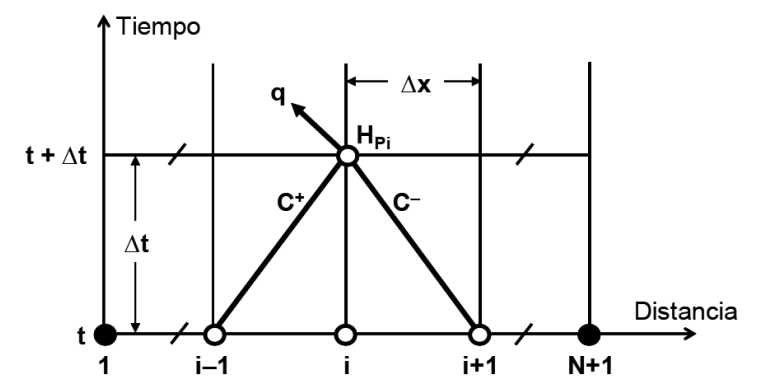

Figura 3: Ecuaciones características con demanda $q$ en el nodo interno $\mathrm{i}$

\section{Resultados}

\section{Ejemplo 1}

La red ejemplo, mostrada en la Figura 4, consta de tres tuberías en serie (T1, T2 y T3) ubicadas entre un reservorio D1 (aguas arriba) y una válvula de regulación Rg1 (aguas abajo), la cual se supondrá inactiva y completamente abierta durante la simulación del transiente. Todas las tuberías tienen $D=200 \mathrm{~mm}, f=0.018$ y $a=1000 \mathrm{~m} / \mathrm{s}$. Sus longitudes son $500 \mathrm{~m}$ (T1), $400 \mathrm{~m}$ (T2) y $600 \mathrm{~m}$ (T3). El caudal inicial es $75 \mathrm{l} / \mathrm{s}$. En orden a obtener $C_{\mathrm{n}}=1.0$ para todas las tuberías, se adoptó un $\Delta t=0.1 \mathrm{~s} \mathrm{y} N=5,4$ y 6 para T1, T2 y T3, respectivamente. El tiempo máximo de simulación es $\mathrm{T}_{\max }=10 \mathrm{~s}$. El flujo transiente es generado por la aparición súbita de una DUD de magnitud $q=61 / \mathrm{s}$ en el tramo T2. En este ejemplo y en el siguiente ejemplo 2 , se supondrá que las demandas nodales son insensibles a la presión, y que el factor de fricción es constante (distinto de transiente). En este último caso el error en las presiones debiera quedar acotado, situación que se presenta cuando el caudal externo total aportado por las demandas $q$ es aproximadamente inferior al $30 \%$ del flujo medio de las tuberías afectadas. La Figura 5 muestra las presiones en función del tiempo en los nodos internos n2-2, n3-2 y n4-2 del tramo $\mathrm{T} 2$.

En la notación el término "n" significa nodo interno, el primer valor numérico identifica la numeración del nodo interno y el segundo valor numérico identifica la numeración del tramo. Por ejemplo, n2-2 significa nodo interno 2 del tramo T2.

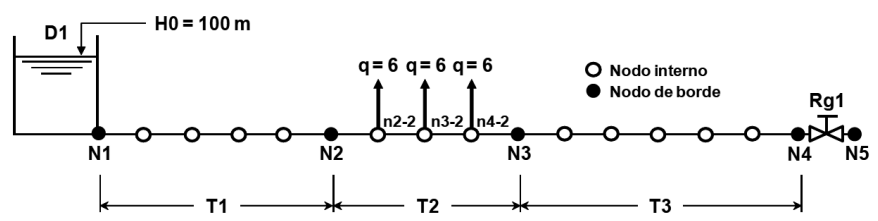

Figura 4: Esquema de la red ejemplo 1 con una DUD $(q=61 / \mathrm{s})$ en el tramo T2. Las demandas se localizan en los nodos internos $\mathrm{n} 2-2, \mathrm{n} 3-2$ y n4-2

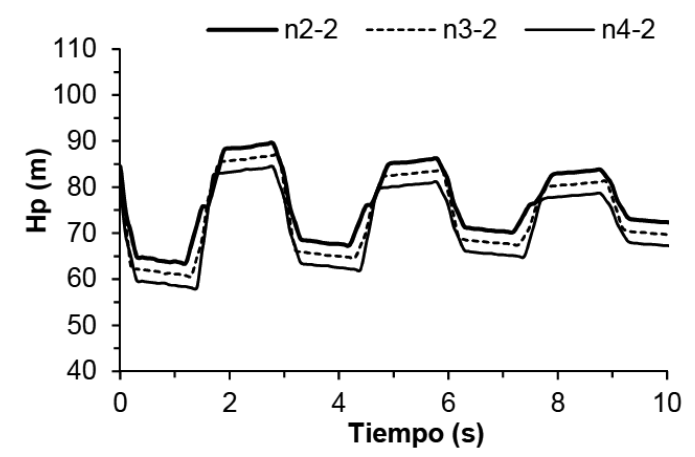

Figura 5: Presión Hp en función del tiempo en los nodos internos $\mathrm{n} 2-2, \mathrm{n} 3-2$ y n4-2 cuando el tramo T2 tiene una DUD con $q=6$ $1 / \mathrm{s}$

\section{Ejemplo 2}

La red de la Figura 6 consta de 7 tuberías (T1, T2, etc.); dos reservorios (D1, D2); 1 válvula de regulación (Rg1) ubicada en el extremo aguas abajo de T7, que descarga hacia la atmósfera y que se supondrá completamente abierta durante la simulación del transiente; 1 válvula aliviadora de presión en N6 (que se supondrá desactivada); 2 nodos (N2 y N5) con una demanda constante $q_{0}$ de 2000 y 1000 l/s, respectivamente; y una chimenea de equilibrio en N3 (Karney y McInnis, 1992).

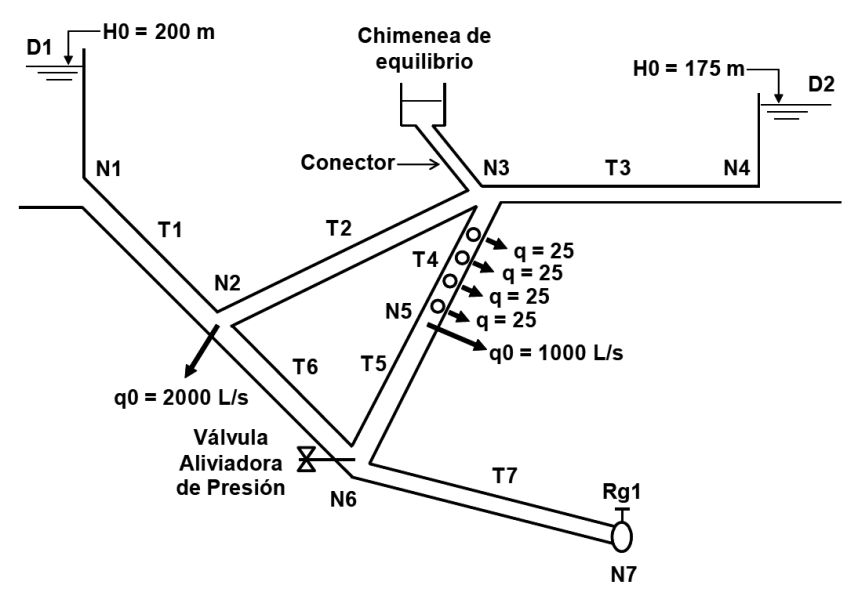

Figura 6: Esquema de la red ejemplo 2 con una DUD con $q=25$ $1 / \mathrm{s}$ en el tramo T4 
La Tabla 1 muestra los datos de las tuberías y de la discretización adoptada en este caso cuando $\Delta t=0.1 \mathrm{~s}$. El transiente es generado por la aparición súbita de una DUD de magnitud $q=25 \mathrm{l} / \mathrm{s}$ en el tramo T4. La Tabla 2 muestra los datos de los nodos. El tiempo máximo de simulación es $\mathrm{T}_{\max }=50 \mathrm{~s}$. La Figura 7 muestra las presiones en función del tiempo en los nodos internos n2-4, n3-4, n4-4 y n5-4 del tramo T4.

Tabla 1: Datos de las tuberías

\begin{tabular}{|c|c|c|c|c|c|c|c|c|}
\hline $\begin{array}{c}\text { Tubería } \\
\mathrm{N}^{\circ}\end{array}$ & $\begin{array}{c}\text { Diámetro } \\
D, \mathrm{~mm}\end{array}$ & $\begin{array}{c}\text { Longitud } \\
L, \mathrm{~m}\end{array}$ & $\begin{array}{c}\text { Número de } \\
\text { subtramos } \\
\mathrm{N}\end{array}$ & $\begin{array}{c}\text { Caudal } \\
\text { inicial } \\
q_{0}, 1 / \mathrm{s}\end{array}$ & $q, 1 / \mathrm{s}$ & $\begin{array}{c}\text { Factor de } \\
\text { fricción } \\
f \text { (Darcy) }\end{array}$ & $\begin{array}{c}\text { V. onda } \\
a, \mathrm{~m} / \mathrm{s}\end{array}$ & $C_{\mathrm{n}}$ \\
\hline $\mathrm{T} 1$ & 1500 & 1001.2 & 10 & 6212 & 0.0 & 0.012 & 996.3 & 1.00 \\
\hline $\mathrm{T} 2$ & 1000 & 2000.0 & 20 & 1708 & 0.0 & 0.013 & 995.3 & 1.00 \\
\hline $\mathrm{T} 3$ & 750 & 2000.0 & 20 & 1183 & 0.0 & 0.014 & 995.0 & 1.00 \\
\hline $\mathrm{T} 4$ & 500 & 502.5 & 5 & 524 & 25.0 & 0.015 & 1000.0 & 1.00 \\
\hline $\mathrm{T} 5$ & 500 & 502.5 & 5 & 476 & 0.0 & 0.015 & 1000.0 & 1.00 \\
\hline $\mathrm{T} 6$ & 1000 & 1001.2 & 10 & 2503 & 0.0 & 0.014 & 996.3 & 1.00 \\
\hline T7 & 750 & 2000.2 & 20 & 2028 & 0.0 & 0.013 & 995.1 & 1.00 \\
\hline Conector & 2523 & 30 & --- & 0 & --- & 0.020 & --- & -- \\
\hline
\end{tabular}

Tabla 2: Datos de los nodos

\begin{tabular}{|c|l|c|c|c|}
\hline $\begin{array}{c}\text { Nodo } \\
\mathrm{N}^{\circ}\end{array}$ & Tipo de nodo & $\begin{array}{c}\text { Elevación } \\
z, \mathrm{~m}\end{array}$ & $\begin{array}{c}\text { Cota } \\
\text { piezométrica } \\
\mathrm{H}_{0}, \mathrm{~m}\end{array}$ & $\begin{array}{c}\text { Descarga } \\
q_{0}, 1 / \mathrm{s}\end{array}$ \\
\hline $\mathrm{N} 1$ & $\begin{array}{l}\text { Reservorio de cota } \\
\text { constante }\end{array}$ & 150 & 200.0 & 0 \\
\hline $\mathrm{N} 2$ & Demanda constante & 100 & 195.0 & 2000 \\
\hline N3 & Chimenea de equilibrio & 150 & 188.8 & 0 \\
\hline N4 & $\begin{array}{l}\text { Reservorio de cota } \\
\text { constante }\end{array}$ & 150 & 175.0 & 0 \\
\hline N5 & Demanda constante & 100 & 183.4 & 1000 \\
\hline N6 & $\begin{array}{l}\text { Válvula aliviadora de } \\
\text { presión }\end{array}$ & 50 & 187.9 & 0 \\
\hline N7 & Válvula de control & 25 & 151.9 & 0 \\
\hline
\end{tabular}

\section{Comparación con el Método de la Repartición Media MRM}

El Método de la Repartición Media MRM es una técnica que determina que la demanda en cada nodo debe corresponder a la sumatoria del $50 \%$ de las demandas propias calculadas para cada tubería. En términos simples, esto significa que, si en la línea de una tubería existen 5 demandas de agua de 1 1/s cada una, entonces el MRM asignará el $50 \%$ de la demanda total, esto es: $0.5 \cdot 5=2.5$ 1/s, a cada nodo de borde de la tubería. En el tramo T2 del ejemplo 1, esto significaría asignar una demanda de

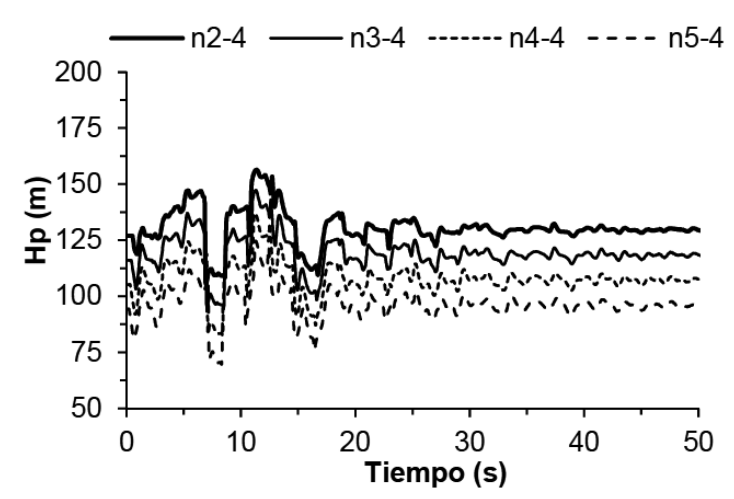

Figura 7: Presión Hp en función del tiempo en los nodos internos $\mathrm{n} 2-4, \mathrm{n} 3-4, \mathrm{n} 4-4$ y n5-4 cuando el tramo T4 tiene una DUD con $q=251 / \mathrm{s}$

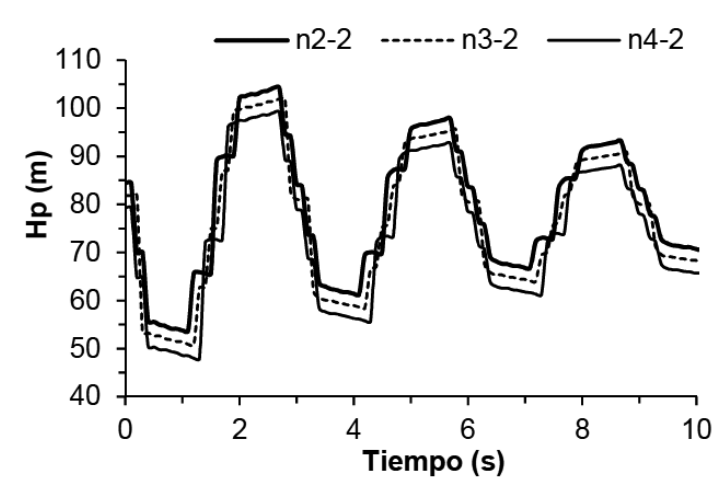

Figura 8: Presión Hp en función del tiempo en los nodos internos n2-2, n3-2, n4-2 del tramo T2 (ejemplo 1) cuando la DUD con $q=6 \mathrm{l} / \mathrm{s}$ es dividida en dos y asignada a los nodos N2 (9 l/s) y N3 (9 1/s)

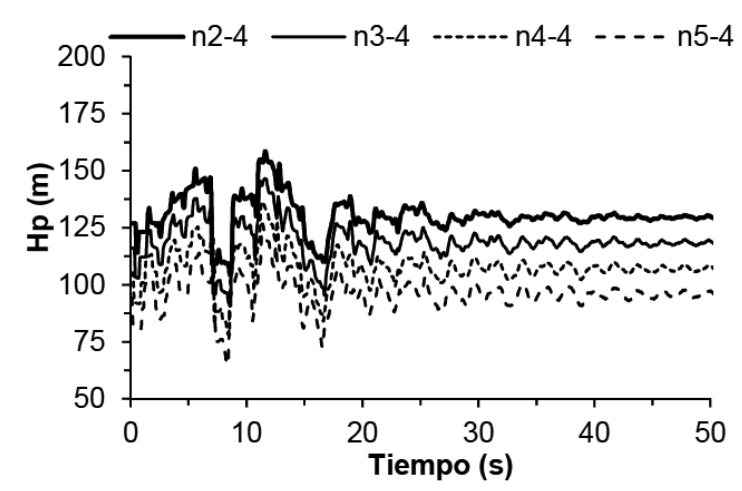

Figura 9: Presión Hp en función del tiempo en los nodos internos n2-4, n3-4, n4-4 y n5-4 del tramo T4 (ejemplo 2) cuando la DUD con $q=25 \mathrm{l} / \mathrm{s}$ es dividida en dos y asignada a los nodos N3 (50 $1 / \mathrm{s})$ y N5 (50 1/s)

9 1/s a los nodos N2 y N3, respectivamente. La Figura 8 muestra la curva de presión en función del tiempo en los nodos internos n2-2, n3-2 y n4-2 de la tubería T2 cuando la demanda en dichos nodos es repartida en una proporción de 
$50 / 50(9 / 9$ l/s) en los nodos N2 y N3. En el tramo T4 de la red ejemplo 2, la asignación de $q=25$ 1/s en los nodos N3 y $\mathrm{N} 5$, respectivamente, significaría asignarles una demanda igual a 50 1/s. La Figura 9 muestra la curva de presión en función del tiempo en los nodos internos n2-4, n3-4, n4-4 y n5-4 de la tubería T4 cuando la demanda en dichos nodos es repartida en una proporción de 50/50 (50/50 1/s) en los nodos N3 y N5.

\section{Discusión}

Se muestra la aplicabilidad de un algoritmo numérico original basado en el MC que permite asignar, a cualquier tubería de una red, una demanda uniformemente distribuida (DUD). El algoritmo es rápido, preciso, explícito y fácil de programar. Por ejemplo, el tiempo de ejecución en los ejemplos 1 y 2 fue inferior a los $1.5 \mathrm{~s}$ al correr el algoritmo en un PC estándar con velocidad de procesamiento de $1.6 \mathrm{GHz}$. Además, la aplicación del algoritmo propuesto supone un aumento marginal de la memoria computacional toda vez que funciona mediante el MC estándar levemente modificado mediante la adición del parámetro $(a / g A) q / 2$ en la ecuación clásica que permite calcular Hpi $(i=2, \ldots$, $\mathrm{N}$ ), siendo $q$ un vector de tamaño proporcional a N. El algoritmo en cuestión tiene la particularidad de que impide tener que re-discretizar la red cada vez que se requiera simular la aparición de una DUD de magnitud $q$ que abarque los nodos internos de una o más tuberías, con lo cual evita tener que definir condiciones de borde adicionales que obligarían a crear y re-numerar nuevos nodos de borde y tramos, lo cual podría constituir una tarea engorrosa en la medida que se incorporan más DUDs al sistema. La DUD puede ser aplicada simultáneamente a uno o más tramos del sistema, sin importar las condiciones de borde que tengan en sus nodos extremos toda vez que la DUD se aplica sólo a los nodos internos de cada tubería. El algoritmo es versátil porque permite modificar la magnitud de la DUD mediante dos vías: (i) incrementando (o decreciendo) el tamaño de $N$, lo cual implicaría la adición (o substracción) de nodos internos con $q$; (ii) variando en mayor o menor medida el valor de $q$, esto último modificando el valor que tenga asignado en una o varias tuberías en particular. Sin embargo, esta segunda opción es más eficiente porque cualquier alteración de $N$ podría significar una distorsión de la discretización inicial, llevando a una situación donde $C_{\mathrm{n}}$ dejaría de ser igual a 1.0 en algunas tuberías, con el consiguiente impacto en la calidad numérica de los resultados. En el ejemplo 1 se verifica que la asignación de las demandas en los nodos de borde de las tuberías con valores iguales al 50\% de la suma de los $q$ (DUD) genera soluciones más conservadoras (ver Tabla 3), tanto para las presiones máximas como mínimas, con diferencias absolutas de $14.2 \%$ y $21.3 \%$, respectivamente. En el ejemplo 2 también se verifica que la demanda nodal es más conservadora que la DUD, aunque en este caso la diferencia absoluta entre las presiones máximas dadas por al DUD y por la demanda nodal es de sólo 1.4\%.

Tabla 3: Comparación de presiones Hp (m) extremas entre DUD y demandas en los nodos de borde de las tuberías

\begin{tabular}{|c|c|c|c|c|}
\hline \multirow{2}{*}{$\begin{array}{c}\text { Ejemplo } \\
\mathrm{N}^{\circ}\end{array}$} & \multicolumn{4}{|c|}{ Tipo de demanda } \\
\cline { 2 - 5 } & Hp máxima & Hp mínima & Hp máxima & Hp mínima \\
\cline { 2 - 5 } & 89.5 & 58.1 & 104.3 & 47.9 \\
\hline 1 & 156.5 & 69.8 & 158.8 & 62.3 \\
\hline 2 & & & & \multicolumn{2}{c|}{ Nodal } \\
\hline
\end{tabular}

Al comparar las presiones mínimas, la diferencia aumenta a $12.0 \%$. Estas diferencias entre los resultados entregados por la DUD y la demanda nodal pueden deberse a que la técnica de repartición media (50/50) implica simular el transiente con demandas de mayor magnitud localizadas en los nodos de borde de las tuberías analizadas. Pese a esto se debe tener presente que la ubicación de las demandas en los nodos de borde de una tubería en la proporción 50/50 puede constituir una regla inadecuada, especialmente cuando la demanda está concentrada en alguno de sus nodos y representa un porcentaje importante del caudal total de la línea (Izquierdo et al., 2013). Otra desventaja relevante del algoritmo es que su funcionamiento óptimo depende de la discretización adoptada para la red, la cual debe ser establecida de forma tal que pueda cumplir con la condición de Courant y, dependiendo del caso, ésta debe ser tal que permita incorporar los nodos internos de interés dentro del análisis. Además, el algoritmo supone que la DUD es insensible a la presión en los nodos internos de las tuberías, lo cual puede llevar a sobreestimar (o subestimar) las condiciones transientes, con riesgo de afectar el dimensionamiento o funcionamiento de los artefactos supresores de ondas y, por ende, de los costos asociados (Jung et al., 2009). 


\section{Conclusiones}

Se plantea un algoritmo útil para simular una o más DUDs en redes de tuberías simples o complejas, permitiendo conocer en forma relativamente sencilla lo que sucede cuando se aplica, en forma súbita, una DUD en uno o más tramos del sistema. La DUD podría servir para analizar el impacto transiente generado por la activación de un sistema contra incendios o de riego, teniendo cuidado de que la discretización adoptada para las tuberías involucradas sea tal que permita hacer coincidir la posición del rociador con el nodo correspondiente a $q$. El concepto sobre el cual se sustenta la DUD contiene una serie de supuestos y simplificaciones que tienden a limitar su campo de acción, siendo extensiones obvias del algoritmo: (i) la incorporación del concepto de dependencia de la presión respecto de $q$ (Salgado et al., 1994; Wu y Walski, 2006); (ii) la inclusión en el modelo de la correlación cruzada (interdependencia) entre demandas próximas (Filion et al., 2007); (iii) la posibilidad de aplicar $q$ sólo en algunos nodos internos de la tubería previamente escogidos por el usuario; y (iv) disponer de otras formas de $q$ asimétricas, distintas de las formas uniformes, con mayores o menores demandas en algunos nodos internos de las tuberías.

\section{Referencias}

Chaudhry, M.H. (2014). Transient-flow equations. In Applied Hydraulic Transients. Springer, New York, USA, 35-64

Chaudhry, M.H. (1979). Applied hydraulic transients. Van Nostrand Reinhold, New York, USA

Chaudhry, M.H. (1968). Boundary conditions for analysis of waterhammer in pipe systems. Thesis of Applied Science, The University of British Columbia, Canada

Filion, Y., Adams, B. and Karney, B. (2007). Cross correlation of demands in water distribution network design. Journal of Water Resources Planning and Management 133(2), 137-144

Gad, A.A. and Mohammed, H.I. (2014). Impact of pipe network simplification on water hammer phenomenon. Sădhana 39(5), 1227-1244

Ghidaoui, M.S., Zhao, M., McInnis, D.A. and Axworthy, D.H. (2005). A review of water hammer theory and practice. Applied Mechanics Reviews 58, 49-76
Izquierdo, J., Campbell, E., Montalvo, I., Pérez-García, R. and Ayala-Cabrera, D. (2013). Error analysis of some demand simplifications in hydraulic models of water supply networks. Abstract and Applied Analysis 2013, article ID 169670, 1-13

Jung, B.S., Boulos, P.F. and Wood, D.J. (2009). Effect of pressure-sensitive demand on surge analysis. Journal of the American Water Works Association 101(4), 100-111

Jung, B.S., Boulos, P.F. and Wood, D.J. (2007). Pitfalls of water distribution model skeletonization for surge analysis. Journal of the American Water Works Association 99(12), 87-98

Kanakoudis, V. and Gonelas, K. (2015). Accurate water demand spatial allocation for water networks modelling using a new approach. Urban Water Journal 12(5), 362-379

Karney, B.W. (1984). Analysis of fluid transients in large distribution networks. $\mathrm{PhD}$ thesis, University of British Columbia, Vancouver, Canada

Karney B.W and McInnis D. (1992). Efficient calculation of transient flow in simple pipe networks. Journal of Hydraulic Engineering 118(7), 1014-1030.

McInnis, D. and Karney, B.W. (1995). Transients in distribution networks: field tests and demand models. Journal of Hydraulic Engineering 121(3), 218-231

Mohammed, H.I. and Gad, A.A. (2012). Effect of pipe network simplification on water hammer phenomenon. Journal of Engineering Sciences 40(6), 1625-1647

Rao, R.M., Ahmed, Z., Ellamraj, Y. and Reddy, R.M. (2015). EPAnet demand calculation methods and implications. International Journal of Scientific Engineering and Research (IJSER) 4(11), 73-76

Salgado, R., Rojo, J. and Zepeda, S. (1994). Extended gradient method for fully non-linear head and flow analysis in pipe networks. Integrated Computer Applications in Water Supply, Methods and Procedures for Systems Simulation and Control. B. Coulbeck (ed.), John Wiley \& Sons, Inc. NY, USA. vol. 1, 49-60

Salgado, R., Twyman, C. and Twyman, J. (1992). Development of a hybrid algorithm for fast transient analysis in pipe networks. XV Congreso Latinoamericano de Hidráulica IAHR, 483-493

Shamrukh, M. (2005). Applicability of using the multiple lateral demands for designing water networks. First Ain Shams University International Conference on Environmental Engineering, Cairo, Egypt, 562-572 
Tsakiris, G. and Spiliotis, M. (2012). Applying resilience indices for assessing the reliability of water distribution systems. Water Utility Journal 3, 19-27

Twyman, J. (2018). Transient flow analysis using the method of characteristics MOC with five-point interpolation scheme.

Obras y Proyectos 24, 62-70

Twyman, J. (2017). Water hammer analysis in a water distribution system. Ingeniería del Agua 21(2), 87-102

Twyman, J. (2016a). Golpe de ariete en una red de distribución de agua. XXVII Congreso Latinoamericano de Hidráulica IAHR Spain Water and IWHR China, Lima, Perú

Twyman, J. (2016b). Wave speed calculation for water hammer analysis. Obras y Proyectos 20, 86-92

Watters, G.Z. (1984). Analysis and control of unsteady flow in pipelines. Butterworth, 2nd edition, Boston, USA
Watters G.Z. (1979). Modern analysis and control of unsteady flow in pipelines. Ann Arbor Science. Michigan, USA

Wu, Z.Y. and Walski, T. (2006). Pressure dependent hydraulic modelling for water distribution systems under abnormal conditions. IWA World Water Congress and Exhibition, Beijing, China

Wylie, B. (1986). Liquid transient flow in piping systems. In Advancements in Aerodynamics, Fluid Mechanics and Hydraulics, ASCE, Arndt, R.E., Stefan, H.G., Farell, C. and Peterson, S.M. (eds.), 50-57

Wylie, B. and Streeter, V.L. (1993). Fluid transients in systems. Pearson. 1st. edition

Wylie, B. and Streeter, V.L. (1978). Fluid transients. McGrawHill, International Book Company 